\title{
Optimized P2P Streaming for Wireless Distributed Networks ${ }^{\text {th }}$
}

\author{
Leonardo Maccari, Nicolò Facchi, Luca Baldesi, Renato Lo Cigno ${ }^{1}$
}

\begin{abstract}
The future Internet will support pervasive applications and communications models that require end-nodes cooperation, such as fog computing and machineto-machine communications. Among the many applications, also video streaming can be provided with a cooperative and peer-to-peer approach. Cooperative distribution requires building a distribution overlay on top of the physical topology (the underlay). This work proposes an optimized, cross-layer approach to build this overlay minimizing the impact on the underlay. We design an optimal strategy, which is proven to be NP-complete, and thus not solvable with a distributed, lightweight protocol. The optimal strategy is relaxed exploiting the knowledge on the betweenness centrality of the nodes in the underlay topology, obtaining two easily implementable solutions applicable to any link-state protocol for distributed wireless mesh networks. The additional introduction of heuristic improvements further optimizes the performance in real network scenarios. Extensive simulation results support the theoretical findings using three different network topologies. They show that the relaxed implementations are reasonably close to the optimal solution, and provide vast gains compared to the traditional overlay topologies that peer-to-peer applications build.
\end{abstract}

Keywords: P2P video streaming, Mesh networks, ad-hoc networks

\section{Introduction}

Fog computing, machine-to-machine communications, Direct WiFi and LTE, the Internet of Things are all indicating that the future trend of the Internet evolution is toward decentralized systems, where peer-to-peer communications will play a mayor role, if not for else, to relieve the backbones and data-centers that are the bottleneck of today centralized architectures. Decentralization

\footnotetext{
This work was partially financed by the European Commission, H2020-ICT-2015 Programme, Grant Number 688768 'netCommons' (Network Infrastructure as Commons).

${ }^{1}$ Department of Information Engineering and Computer Science (DISI), University of Trento, Italy - \{leonardo.maccari,nicolo.facchi,luca.baldesi,renato.locigno\}@unitn.it
} 
for sustainability is probably one of the key factors that will enable pervasive computing and communications.

Streamlined communications, video but not only, will remain among the largest bandwidth guzzlers, as they are today, and their efficient support is of paramount importance for the scalability of the entire system. Currently, the majority of the Video Service Providers (VSPs) deliver video streams using unicast traffic and leveraging centralized platforms supported by world-wide Content Delivery Networks (CDNs). A CDN is a very costly resource that replicates the content as close to the final user as possible. CDNs are approximately co-located with Internet exchange points and replicate videos based on the estimated popularity of the video itself. Users access replicas cached in CDNs, so that the total load on the VSP infrastructure is limited. This approach does not fit with the advent of a communication model in which a multitude of video flows and real-time streams are generated by the user's smart-house, his/her friends, co-workers, relatives, then sent upstream to a cloud server and finally streamed on the user's device. Each stream is useful for a small number of people, so the convenience of CDNs and cloud-based caching vanishes. Pervasive computing is based on a distributed mesh of wireless (or wired) devices, which form an underlay network over which live video and streams are produced, served from the local generating device and disseminated globally. An already relevant example of this trend is represented by Community Networks (CNs). CNs are large mesh networks (primarily made of wireless links) that are flourishing in many different scenarios, from developing countries where there are no other connectivity means, to urban areas of Western cities where they complement (or compete with) traditional network providers. The steep decrease of the prices of outdoor wireless equipment makes it possible to build wireless mesh networks with links that can achieve tens of Mbit/s and support CNs made of hundreds of nodes. Today, CNs made of thousands of devices that cover large cities or even regions exists, like Guifi in Spain or Freifunk in Germany ${ }^{2}$. These networks already connect with a peer-to-peer (P2P) paradigm thousands of people, and potentially, tomorrow, hundreds of sub-networks made of smart devices.

In this perspective, the $\mathrm{P} 2 \mathrm{P}$ paradigm is rejuvenating its actual application in real systems. Despite a very large interest from the academia, indeed, in the last 15 years P2P applications could not compete with centralized, cloudbased applications. The key factor hampering P2P development, specially for video distribution, has been the difficulty to realize $\mathrm{P} 2 \mathrm{P}$ overlays optimized from the point of view of the Internet Service Providers (ISPs), mostly due to lack of information on the physical network. In mesh networks, the underlay is normally known, since the routing protocols exports it to each node (as long as a link-state routing protocol is used), which removes one of the technical barriers that blocked the deployment of P2P video streaming on the Internet.

In this paper, we propose a cross-layer optimization scheme to perform live video streaming (i.e., with a strict deadline on the arrival delay) in mesh net-

\footnotetext{
${ }^{2}$ See http://www.guifi.net and http://www.frefunk.org
} 
works [1]. The optimization minimizes the impact of the streaming overlay on the underlay network exploiting information on the topology and the routing of the underlay. The optimization is based on the concept of centrality, which is also at the base of successful algorithms as Google PageRank [2]. Taking into account the centrality of peers in the underlay graph, the optimized overlay topology greatly improves the efficiency of the video distribution and maintains high performance.

More specifically, our key contributions are the following ones:

- We formalize an optimization problem that, given the underlay network and the peers in the overlay, finds the overlay topology whose cost on the underlay is minimum. We define the cost of the overlay as a combined metric composed by the load and the fairness imposed by the overlay on the underlay;

- We prove that the optimal strategy is NP-complete by reducing it to a quadratic knapsack problem;

- We propose two techniques for relaxing the optimal strategy that relies on the concept of betweenness centrality of the nodes of the underlay. The relaxed strategies are applicable to any wireless mesh network, as long as they use a link-state routing protocol;

- We further improve the performance of the relaxation strategies by introducing a neighborhood pruning heuristic which exploits characteristics often found in real network scenarios;

- We evaluate the proposed relaxation strategies and pruning heuristics through extensive simulations that compute the best overlay, according to each proposed technique, on synthetic network topologies. Simulation results show that the proposed relaxations and heuristics are reasonably close to the optimal solution and largely outperform random overlay building strategies.

This paper builds on a previous work in which we formalized the optimization problem and, introduced the relaxation strategy and evaluated its results [3]. This paper extends these results with further optimizations to make the proposal more efficient in overlays with a realistic topology, i.e. comprising many leafnode in the network graph.

The rest of this paper is organized as follows: in Section 2 we formalize the optimization problem. The related work on this area is summarized in Section 3. Section 4 introduces the intersection graph notation we use for defining the overlay. In Section 5 we describe the strategies used for relaxing the optimization problem, and the heuristic used for further improving the relaxation strategies is described in Section 6. We analyze the performance of the proposed strategies and heuristics in Section 7. Finally, Section 8 concludes the paper. 


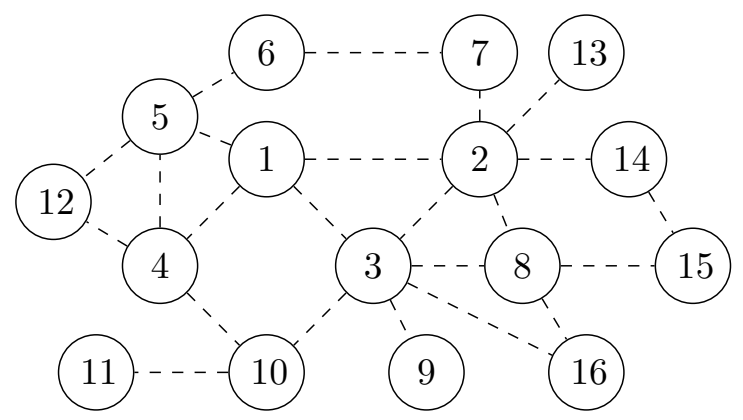

Figure 1: Example of wireless mesh underlay graph. Hosts are numbered with an arbitrary ordering. Dashed lines represent the wireless links.

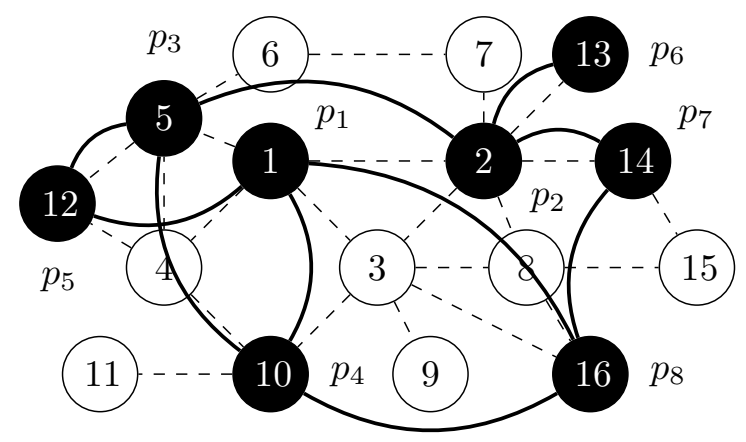

Figure 2: Possible overlay graph (black vertexes and edges) over the underlay graph of Fig. 1.

\section{Motivation and Problem Statement}

We consider a communication system where the cooperative distribution exploits a logical topology called the overlay built on top of a meshed routing network called the underlay. Nodes in the overlay, that from now on we call peers, do have access to information concerning the underlay, including details on the topology and quality of its links. This is true for a wireless mesh network using link-state routing protocols, in which every wireless router knows the whole topology of the network to compute routes with Djikstra algorithm. For instance, the OLSRd daemon that implements the Optimized Link State Routing (OLSR) protocol [4] exports the topology with a simple API. The optimization we propose regards the choices of the edges in the overlay so that the impact on the underlay is minimized and evenly distributed.

We model the underlay with an undirected graph $U(H, L)$ with vertexes $h \in H$ called hosts or nodes, and edges $l \in L$ called links. The size of $H$ is between a few tens up to a thousand hosts, which corresponds to the realistic size of a CN [5]. Fig. 1 shows the graph representation of a sample underlay with 16 hosts. 
Table 1: Summary of the main symbols used through the paper and their meaning.

\begin{tabular}{l|r} 
Peers, Hosts, Links and Virtual Links sets & $P, H, L$, and $E$ \\
\hline Overlay and underlay graphs & $U(H, L), O(P, E)$ \\
\hline Fairness of $O(P, E)$ over $U(H, L)$ & $\mathcal{F}$ \\
\hline Network load of $O(P, E)$ over $U(H, L)$ & $\mathcal{L}$ \\
\hline$k$ th undirected overlay edge in $P \times P$ & $e_{k}$ \\
\hline Cross-layer overlay edge descriptor of $e_{k}$ & $\bar{e}_{k}$ \\
\hline$i$ th overlay peer & $p_{i}$ \\
\hline Degree of $p_{i}$ in the overlay & $d_{i}$ \\
\hline Set of the overlay edges in $O(P, E)$ linking $p_{i}$ & $S_{i}^{\prime}$ \\
\hline Family of all $S_{i}^{\prime}, i=1, \ldots,|P|$ & $F^{\prime}$ \\
\hline$O(P, E)$ as an intersection graph & $\Omega\left(F^{\prime}\right)$ \\
\hline Target function on $\Omega\left(F^{\prime}\right)$ addressing $\mathcal{L}$ and $\mathcal{F}$ & $O_{c}$ \\
\hline Binary variable representing of whether $e_{k} \in E$ & $z_{k}$ \\
\hline Estimation of link usage in $O(P, E)$ & $b$
\end{tabular}

The peers form an overlay that is also modelled as an undirected graph $O(P, E)$ with vertexes $p \in P$ called peers, and edges $e \in E$ called virtual or logical links. Each peer resides in one host only, and it has access to information pertaining to $U$, including the association between peers and hosts. Fig. 2 depicts a possible overlay graph on the underlay of Fig. 1.

The goal is to find a viable (meaning that can be implemented as a distributed system with limited signaling overhead and acceptable computational overhead) methodology to select virtual links between peers to build $O(P, E)$ given $U(H, L)$ and $P$ so that the load imposed by the video streaming on the underlay links is minimized, and links are loaded as fairly as possible. $O$ is dynamically created and maintained because both $O$ and $U$ can change frequently, so the modification of $O$ must be fast and efficient.

\subsection{Formal Problem Definition}

Tab. 1 reports the main notation we use in the paper. Given an edge $e$ connecting $p_{i}, p_{j} \in P$, we call $\mathrm{D}(e)$ the Dijkstra function returning an (ordered) set of links in the underlay that form the shortest path from the host where $p_{i}$ resides and the host where $p_{j}$ resides. For example in Fig. 2 we have:

$$
e=\left(p_{1}, p_{4}\right) \mapsto \mathrm{D}(e)=\{(1,3),(3,10)\}
$$

where $(i, j)$ is the link $e$ connecting hosts $i$ and $j$. A generic weight $w(l)$ is assigned to any link in the underlay, so that the load $\mathcal{L}$ imposed by $O$ on $U$ is

$$
\mathcal{L}=\sum_{e \in E} \sum_{l \in \mathrm{D}(e)} \frac{w(l)}{s(e)}
$$

The $s(e)$ denominator represents a scaling factor for the edge, we detail it and use it in rest of the paper to express the fact that some edges carry more 
traffic than others. This representation perfectly fits the routing protocols that use the ETX metric [6], ETX is the expected average number of frames sent on the link to correctly deliver one frame, and it is used by OLSR and other protocols.

Every link $l$ is loaded by a number of virtual links. To measure fairness, we use Jain's fairness index on the distribution of the number of logical links insisting on every $l$. Let $\mathcal{H}(l)$ be the number of logical links loading $l$ :

$$
\mathcal{H}(l)=|\{e \in E: l \in \mathrm{D}(e)\}|
$$

where $|\cdot|$ is the size of a set. The Jain's fairness is defined as

$$
\mathcal{F}=\frac{\left(\sum_{l \in L} \mathcal{H}(l)\right)^{2}}{|L| \sum_{l \in L} \mathcal{H}(l)^{2}}
$$

Jain's fairness is maximal if $\mathcal{F}=1$ and minimal when $\mathcal{F}=\frac{1}{|L|}$, but we do not expect that maximal fairness can be reached, as in general there are links in the underlay that do not support any edge in the overlay.

The contribution we present in the sequel, after discussing the related work follows two lines:

- First we derive a formal framework wherein it is possible to define an optimization problem that allows finding the topology of $O(P, E)$ given $U(H, L)$ and $P$ that minimizes a metric composed of $\mathcal{L}$ and $\mathcal{F}$. The problem is NP-complete and we'll show that it can be reduced to a quadratic knapsack problem [7];

- Second we propose two relaxations of the optimization in decreasing complexity order and we show, with numerical solutions and with an implementation in a real P2P streaming platform, that the two relaxations are close to the global optimum and that they vastly outperform the traditional P2P overlay building based on selecting uniformly neighboring peers to build an Erdös-Rényi graph.

The paper focuses on building a mesh overlay and not on how video chunks are distributed on it. On mesh topologies this latter problem can be tackled with several different strategies. Even if this is not the focus of the paper, Sec. 3 briefly refers some relevant works on this topic, justifying our choice for the distribution strategy we use in Sec. 7 and Sec. 7.1. The assumption we start from, which becomes a constraint of the optimization problem, is that each peer should receive exactly one copy of every chunk. In our previous work [3] we also assumed that each peer contributes to the dissemination serving chunks to other peers proportionally to its degree in $O$. This was motivated by the effort to keep the number of neighbors per peer constant (with a quasi-regular topology), so that the overall load was fairly distributed. In this paper we introduce a new model, in which we assume that every peer will generate a constant amount of traffic, evenly distributed among its neighbors. This way we support non quasi-regular topologies that match better with the underlay topologies. 
The work presented in [3] used two evaluation strategies: graph analysis with Matlab, and the Python Networkx graph library. Results were validated implementing the strategies on a real P2P video streaming platform, PeerStreamer [8]. We showed that the first methodology approximates extremely well the real implementation. In this work we focus on further insights on the theoretical model and its validation and thus we rely on the first instrument and we leave the implementation as future work.

\section{Related Works}

Cooperative video streaming (including P2P) is an established research area. We focus on unstructured and mesh-based approaches, in which, differently from structured P2P networks [9], there is no specific structure (like a tree) in the topology. This approach has been shown to be particularly robust even in networks with churn (i.e., peers leaving and joining the swarm), and the overlay topology design is of paramount importance [10].

We do not consider here papers that perform streaming optimization on mesh networks requiring modifications to the lower layers (they cannot be applied to existing CNs), or that are not tailored for live video streaming (e.g., using large chunks that imply several seconds of buffering delay). We also do not consider techniques (e.g., like cloud-assisted or SDN based), where the role of the peers is, in one way or another, not fundamental, and we assume that security [11] and collusion [12] issue need not be solved by the application itself. Finally, we assume all the peers to be static, since handover with standard protocols introduces important delays in mesh networks [13] and customized/optimized solutions are still an open research theme [14]. The following discussion is focused on two parts: topology management, which is directly related to our contribution, and chunk/information scheduling, which justify the choice of the chunk selection strategy in Sec. 7 .

\subsection{Topology Management}

As we already mentioned, overlay optimization on the Internet is not feasible due to lack of information on the underlay details; however several efforts have been done to adapt and improve the overlay topology to some measured underlay characteristics.

The first approach to mention is the use of "network coordinates" as a means to compute distances between hosts in a certain space. Several algorithms were proposed $[15,16,17]$, that are designed to work in the heterogeneous environment of Internet. In all of them the goal is clearly to find a method to infer details on the underlay (the Internet), a problem that we do not have, as we take advantage of the available information on the network topology provided by routing protocols in Wireless Mesh Networks (WMNs). We believe that our solution can be adapted, albeit not straightforwardly, to situations where the underlay is not known but approximated with network coordinates.

A second line of research deals with the adaptation of the overlay based on bandwidth [18] or delay measures $[19,20]$ (normally the round trip time between 
peers), but also on a mix of the two [21, 22]. The solutions found in these works are, once again, tailored to the Internet, where delays can be large (CNs spans a few tens of $\mathrm{km}$ at most), and bandwidth asymmetry at the edges imposes hard limits to the capacity of peers to contribute to dissemination.

Extremely interesting and promising for topology management is the adoption of centrality metrics as means to better understand the topology characteristics of a network graph as it emerges from the routing protocol. Centrality metrics in graphs have been used in social science since the 70 s to identify the most influential elements in social networks. Quite surprisingly, they were not applied to multi-hop networks up to recent times [2]. Centrality metrics can be used to enhance network monitoring and routing [23], as well as the resilience of routing algorithms to networks failures [24] intrusion detection and firewalling $[25,26]$, and topology control [27]. There are several metrics based on different centrality "concepts". In this paper we use the betweenness centrality (see [26] for a definition tailored to our problem) to relax the optimization problem as it is strictly related to shortest path routing.

\subsection{Chunk Scheduling}

In a mesh-based overlay the problem of chunk scheduling is the selection of the neighbors to send/receive information to/from while contextually choosing the right information (chunk) to send/retrieve. This problem has been extensively studied $[28,29,30,31,32]$, and in some specific contexts with restrictive assumptions, the existence of an optimal scheduling strategy has been proven $[33,34]$. These works show that an efficient and robust chunk scheduling technique that works well in most environments consists in selecting a neighbor with a random (possibly weighted) strategy, and push the most recent chunk that is still missing at the receiving peer (Latest Useful Chunk). An idea that has been recently revived and analyzed with the name high-bandwidth peer first $(\mathrm{HPF})$ in $[35]$.

\section{Overlay Model}

The links in $U$ are bidirectional and assumed quasi-symmetric: the most common routing protocols take care of excluding unidirectional or highly asymmetric links. Thus both $O$ and $U$ are undirected, and the maximum number of edges they can have is $m_{O}=\frac{|P|^{2}-|P|}{2}$ and $m_{U}=\frac{|H|^{2}-|H|}{2}$ respectively.

Let $r \in 1 \ldots m_{U}$ be an arbitrary ordering on the links; $r$ is also a mapping from the two hosts $h_{i}$ and $h_{j}$ that are the endpoints of the link: $r\left(h_{i}, h_{j}\right)$. Similarly we define an ordering $k \in 1 \ldots m_{O}$ on the edges of $O$, and $k$ is a mapping $k\left(p_{i}, p_{j}\right)$.

Every link $l_{r}$ is represented by a binary array of size $m_{U}$ with the $r$ th element set to one and all other elements set to zero (we use the bar sign to refer to the array representation of a link):

$$
\bar{l}_{r}=(0, \ldots, 0,1,0, \ldots, 0)
$$




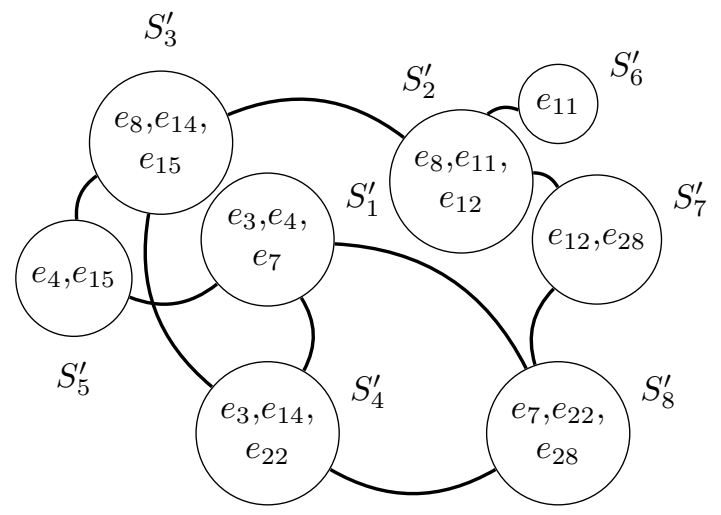

Figure 3: Example of overlay intersection graph. Elements $e_{k}$ are the cross-layer overlay edge descriptors between the nodes.

Equivalently each virtual link $e_{k}$ is represented by the sum of the link arrays in the corresponding shortest path:

$$
\bar{e}_{k}=\sum_{l_{r} \in \mathrm{D}\left(e_{k}\right)} \bar{l}_{r}
$$

We call $\bar{e}_{k}$ the cross-layer overlay edge descriptor, since it bonds the overlay to the underlay. Link weights $w(l)$ in $U$ can be easily taken into account: let $W \in \mathbb{R}^{m_{U} \times m_{U}}$ be a diagonal matrix such that $W_{r, r}=w\left(l_{r}\right)$, then $\bar{e}_{k} W$ is the weighted representation of the overlay edge.

\subsection{Overlay re-definition as an intersection graph}

Given all $\bar{e}_{k}$ for a set of peers $P$, we can take advantage from a transformation into the intersection graph space (see [36] for the complete definitions and properties of intersection graphs) in order to formulate the optimization problem of overlay construction.

Let $S$ be a set and $F=\left\{S_{1}, \ldots, S_{p}\right\}$ a nonempty family of distinct nonempty subsets of $S$ whose union is $S$. The intersection graph of $F$ is denoted $\Omega(F)$, with $S_{i}$ and $S_{j}$ adjacent whenever $i \neq j$ and $S_{i} \cap S_{j} \neq \emptyset$. It is easily shown that if $O(P, E)$ is a full mesh then it is isomorphic with the intersection graph space $\Omega(F)$ where

$$
S_{i}=\left\{\bar{e}_{k\left(p_{i}, p_{j}\right)}, \forall p_{j} \in P\right\} \forall p_{i} \in P ; \quad S=\cup_{i=1}^{|P|} S_{i}
$$

and each $S_{i}$ is the set of all the possible virtual links built on shortest paths from peer $p_{i}$ to all other peers. As a consequence, given an underlay $U(H, L)$ and a set of peers $P$, any overlay $O(P, E)$ over $U(H, L)$ can be defined as the intersection graph $\Omega\left(F^{\prime}\right)$ with $F^{\prime}=\left\{S_{1}^{\prime}, \ldots, S_{|P|}^{\prime}\right\}$ where $S_{i}^{\prime} \subseteq S_{i} \forall S_{i} \in F$. If each peer chooses only a subset $S_{i}^{\prime} \subseteq S_{i}$ and activates only a subset of possible 
edges, then the resulting overlay is isomorphic with some $\Omega\left(F^{\prime}\right)$. The overlay depicted in Fig. 2 is isomorphic with the intersection graph shown in Fig. 3: every $S_{i}^{\prime}$ in Fig. 3 corresponds to a peer $p_{i}$ in Fig. 2.

\subsection{Performance Measures}

We can now redefine in terms of intersection graphs also the performance metrics expressed by Eq. (1) and Eq. (3) in Sec. 2:

$$
\mathcal{L}=O_{I}\left(\Omega\left(F^{\prime}\right)\right)=\overrightarrow{1} \cdot L\left(F^{\prime}\right) ; \quad L\left(F^{\prime}\right)=\sum_{\bar{e}_{k} \in S^{\prime}} \bar{e}_{k} \frac{W}{s\left(e_{k}\right)}
$$

where $L\left(F^{\prime}\right)$ is the array that associates the traffic potentially produced by the overlay to each link in the underlay, $\overrightarrow{1}$ is the array of size $m_{U}$ made of all ones, and $\cdot$ is the dot product. Given the assumption on the traffic generated by every peer that we made in the end of Sec. 2.1 we can set $s\left(e_{k}\right)=\left(d_{i} d_{j}\right) /\left(d_{i}+d_{j}\right)$, where $d_{i}, d_{j}$ are the degrees of the peers that constitute the endpoints of $e_{k}$. This formulation of $s\left(e_{k}\right)$ takes into consideration the distribution of the unitary load generated by a peer on its neighbors. If all the peers have the same degree $s\left(e_{k}\right)$ is constant and can be omitted from the formulation. Else, it takes into account that a peer with many neighbors will load each of its edges less than a peer with few neighbors.

Eq. (5) redefines Eq. (1) through operations done in the intersection graph space. Similarly we re-define the Jain's fairness index as

$$
\mathcal{F}=O_{f}\left(\Omega\left(F^{\prime}\right)\right)=\frac{\left(\sum_{k=1}^{m_{U}} L\left(F^{\prime}\right)_{i}\right)^{2}}{m_{U}\left(\sum_{k=1}^{m_{U}} L\left(F^{\prime}\right)_{i}^{2}\right)}
$$

\section{Overlay Optimization}

In this section we consider regular overlays with constant degree, so we omit $s\left(e_{k}\right)$, and without loss of generality, we take as weighting matrix $W$ the identity matrix. In Sec. 6 we show that regular overlays are suboptimal over some realistic underlays, and show the role of non unitary $W$ in these cases. We want to build an overlay $O$ determined by a choice of $F^{\prime}=\left\{S_{1}^{\prime}, \ldots, S_{|P|}^{\prime}\right\}$ that minimizes the load on the underlying edges and guarantees a fairness as

close as possible to 1 . We have to choose the sets $S_{i}^{\prime} \subseteq S_{i}$ with $S^{\prime}=\cup_{i=1}^{|P|} S_{i}^{\prime}$ such that both $O_{I}$ and $O_{f}$ are minimal, which is a multi-objective combinatorial optimization problem. The problem allows the definition of a combined metric $O_{c}$ that expresses the cost of the overlay. Since each array $\bar{e}_{k} \in S^{\prime}$ corresponds to a set of links the cost of the overlay is defined as:

$$
O_{c}\left(\Omega\left(F^{\prime}\right)\right)=\left\|\sum_{k=1}^{\left|S^{\prime}\right|} \bar{e}_{k}\right\|_{2}
$$


The creation of an efficient overlay $\Omega\left(F^{\prime}\right)$ can be formulated as a minimization problem as follows. Select $F^{\prime}$ in order to minimize the expression:

$$
\arg \min _{z}\left\|\sum_{k=1}^{|S|} z_{k} \bar{e}_{k}\right\|_{2}
$$

where

$$
z_{k}= \begin{cases}1 & \text { if } \bar{e}_{k} \in S^{\prime} \\ 0 & \text { otherwise }\end{cases}
$$

In order to avoid a trivial solution we impose on each peer a minimum node degree $d>\log _{2}(|P|)$, which also guarantees that the resulting overlay is connected with high probability ${ }^{3}$.

This problem can be rephrased as: find the overlay graph $\Omega\left(F^{\prime}\right)$ with minimum degree $d$ defined by $F^{\prime}=\left\{S_{1}^{\prime}, \ldots, S_{|P|}^{\prime}\right\}$ so that the norm of $L\left(F^{\prime}\right)$ is minimized subject to the following constraint

$$
\left(\sum_{\bar{e}_{k} \in S_{i}} z_{k}\right) \geq d ; \quad \forall i=1 \ldots|E|
$$

In Eq. (8) we did a small abuse of notation to improve the readability: the sum spans all the edges $e_{k} \in S_{i}$, but it is indeed a sum over $k\left(p_{i}, p_{j}\right)$ to correctly identify the indication function $z_{k}$. We will use this notation also in several other equations.

If, instead of the norm, in Eq. (7) we use the sum of the elements, this would simply minimize $O_{I}$. The norm instead prefers solutions that are close to the minimum $O_{I}$ and, among two potential solutions with the same $O_{I}$, it prefers the one in which the weights of $L\left(F^{\prime}\right)$ are more fairly distributed.

This problem is a zero-one quadratic programming problem [37], similar to a quadratic knapsack problem [7]. In this kind of problems one wants to minimize the value of an expression $c^{T} x+x^{T} Q x$ where $x=\{0,1\}^{n}$ is an array of binary variables, $c \in R^{n}$ and $Q$ is a symmetric matrix of size $n \times n$. The minimization is subject to a constraint of the kind $h^{T} x+x^{T} G x>g$, where $h$ is an array of size $n, G$ a symmetric matrix of size $n \times n$ and $g$ some real value. If we call $\bar{A}$ the matrix made of columns corresponding to the arrays $\bar{e}_{k}$ and $z$ the array made of $z_{k}$ elements then:

$$
\arg \min _{z}\left\|\sum_{k=1}^{|S|} z_{k} \bar{e}_{k}\right\|_{2}=\arg \min _{z} z^{T} \bar{A}^{T} \bar{A} z
$$

Our problem is thus a zero-one quadratic problem with $Q=\bar{A}^{T} \bar{A}, G$ and $c$ made of all zeros, $h=\overrightarrow{1}$. This family of problems is known to be NP-hard, but there are algorithms in literature that make them tractable up to a certain size

${ }^{3}$ Using simple P2P techniques the probability actually converges to 1 [22]. 
using branch-and-bound techniques [37]. Still, when $|S|$ grows beyond a few hundreds the problem cannot be solved on commodity hardware. $|S|$ corresponds to the number of possible edges in the overlay $m_{O}$, so it scales quadratically with the number of peers, which quickly makes the problem intractable.

\subsection{Betweenness Centrality-based Relaxation}

We need to find a relaxation in which each $p_{j}$ can solve a portion of the problem making some assumptions on the behaviour of the other peers. This corresponds to a scenario in which every peer is aware of the other peers, independently selects its own neighbors, and it communicates them its choice. This is the way P2P streaming protocols based on peer sampling typically work (including the already mentioned PeerStreamer).

Let us first separate the contribution to the overall cost of the edges chosen by $p_{j}$ and all the other peers in Eq. (7)

$$
\left\|\sum_{k=1}^{|S|} z_{k} \bar{e}_{k}\right\|_{2}=\frac{1}{2}\left\|\sum_{S_{i} \in F, S_{i} \neq S_{j}} \sum_{\bar{e}_{k} \in S_{i}} z_{k} \bar{e}_{k}+\sum_{\bar{e}_{k} \in S_{j}} z_{k} \bar{e}_{k}\right\|_{2}
$$

The value $\frac{1}{2}$ comes from the observation that when we separately count each link, every link is counted twice in the sum. Let's call $b_{j}$ the vector representing the choices of the peers in $P \backslash\left\{p_{j}\right\}$ :

$$
\left\|\sum_{k=1}^{|S|} z_{k} \bar{e}_{k}\right\|_{2}=\frac{1}{2}\left\|b_{j}+\sum_{\bar{e}_{k} \in S_{j}} z_{k} \bar{e}_{k}\right\|_{2}
$$

and we can say that:

$$
\arg \min _{z}\left\|\sum_{k=1}^{|S|} z_{k} \bar{e}_{k}\right\|_{2}=\arg \min _{z}\left\|b_{j}+\sum_{\bar{e}_{k} \in S_{j}} z_{k} \bar{e}_{k}\right\|_{2}
$$

The goal is to find a relaxation of the problem where every peer chooses its own neighborhood making some assumptions about $b_{j}$, which represents the choice that the other peers $p_{i} \neq p_{j}$ do. We need to find a reasonable approximation $\bar{b} \simeq b_{j}$ that node $p_{j}$ can use in (11).

Let us introduce a notion that helps in this task. In graph theory, the notion of betweenness centrality is a property of the edges (or of the nodes) of a graph defined as the fraction of the total number of shortest paths that passes through that edge (or node). It is a metric used to identify the edges (or nodes) that are more involved in multi-hop interactions between the vertexes of a graph, so for some applications they can be considered more important than the others.

We call $b=\sum_{\bar{e}_{k} \in S} \bar{e}_{k}$ the summation of all the cross-layer overlay edge descriptors of the complete overlay. Recall that $\bar{e}_{k}$ corresponds to a shortest path in $U$ between two hosts on which a peer resides, thus, each element of $b$ 
corresponds to a link in $U$ and expresses the number of shortest paths in the set $S$ that insist on that link.

Consider the limit case where every host in the underlay contains a peer $(|P|=|H|)$ and let $b^{*}$ be the value of $b$ normalized to the total number of shortest paths:

$$
b^{*}=b \frac{2}{|P|^{2}-|P|}
$$

$b^{*}$ is exactly the vector corresponding to the betweenness centrality of each link in $L$.

If $|H|>|P|, b^{*}$ is an approximation of the real array of centralities. This is a known fact that is used to approximate centrality in large networks: if the number of nodes is too large to compute all the shortest paths, centrality can be estimated using a subset of the paths chosen from a random set of nodes [38]. A key fact is that the convergence to a solution close to the real one is pretty fast in power-law graphs, that are extremely frequent in real communication networks, and also in some large CNs [39]. Thus, even if $p_{j}$ ignores $b_{j}$, a reasonable assumption is that whatever the choice of every other peer is, the elements of $b_{j}$ (that represent the sum of all choices) have a shape similar to the centrality expressed by the normalized value $b^{*}$. This, on power-law underlays is true even for values of $|P|$ one order of magnitude smaller than $|H|$.

Given that we impose each peer to have (at least) $d$ neighbors, the number of edges in $E$ will be approximately $\frac{d|P|}{2}$ and finally the best approximation for $b_{j}$ turns to be

$$
\bar{b}=b^{*}(|P|-1) \frac{d}{2}=b \frac{d(|P|-1)}{|P|^{2}-|P|}=b \frac{d}{|P|}=\frac{d \sum_{k=1}^{|S|} \bar{e}_{k}}{|P|}
$$

The complexity of Eq. (12) is polynomial with $P$ [38], that allows the computations of its solution for overlays of hundreds of peers using commodity hardware. Moreover in communication networks, that are sparse graphs, betweenness can be computed quickly using heuristics [40], which explains why we introduced this formulation based on centrality. Thus, replacing $b_{j}$ with $\bar{b}$ in (11) each peer $p_{j}$ resolves the following optimization problem:

$$
\begin{gathered}
\arg \min _{z}\left\|\bar{b}+\sum_{\bar{e}_{k} \in S_{j}} z_{k} \bar{e}_{k}\right\|_{2} \\
\text { conditioned to: } \sum_{\bar{e}_{k} \in S_{j}} z_{k} \geq d
\end{gathered}
$$

The formulation of (13) is another zero-one quadratic minimization problem, but the dimension of the problem is now bounded by $|P|<|S|$ (the maximum number of neighbors for $p_{j}$ ), and can be effectively solved up to hundreds of peers. In the rest of the paper we will use the branch-and-bound solver given by the YALMLIP library [41] which solves the problem (13) for a network of 100 nodes in a few seconds. 
If the dimension of the problem or the available hardware do not allow the solution of (13), we can apply a greedy search algorithm, ordering edges $\bar{e}_{k}$ on their weight and choosing the ones that minimize the sum. This corresponds to relax (13) to:

$$
\begin{aligned}
& \arg \min _{z} \sum_{\bar{e}_{k} \in S_{j}} z_{k}\left\|\bar{b}+\bar{e}_{k}\right\|_{2} \\
& \text { conditioned to: } \sum_{\bar{e}_{k} \in S_{j}} z_{k} \geq d
\end{aligned}
$$

which of course captures only a part of the original problem, but greatly simplifies the computation. Results show that the solutions generated by (7), (13) and (15) in many realistic network topologies are reasonably close.

It is worth nothing that if we set $\vec{b}=\overrightarrow{0}$ in Eq. (15), then the l-2 norm yields the same order of the l-1 norm. In practice each $p_{j}$ chooses the neighbors that are closer in terms of hops in the underlay. The $\bar{b}$ term instead introduces a bias in the choice towards the neighbors connected through links that are less overloaded and introduce a higher fairness. In the comparison we include also a strategy in which $\vec{b}=\overrightarrow{0}$ because this strategy can be used also in absence of full information from the underlay topology, since the distance from another peer can be measured with probing tool (like the traceroute application) or can be inferred from the TTL field in IP packets. Results show that the performance of this simple ranking function is much lower compared to the proposed strategies. Tab. 2 summarizes the different optimization strategies and labels them with the names we use.

\begin{tabular}{|c|c|c|}
\hline Name & Symbol & Formula \\
\hline Global Optimization & $G_{o}$ & $\arg \min _{z}\left\|\sum_{k=1}^{|S|} z_{k} \bar{e}_{k}\right\|_{2}$ \\
\hline Local Optimization & $L_{o}\left(L_{o p}\right)$ & $\arg \min _{z}\left\|\bar{b}+\sum_{k=1}^{\left|S_{j}\right|} z_{k} \bar{e}_{k}\right\|_{2}$ \\
\hline Local Equalized Ranking & $E_{r}\left(E_{r p}\right)$ & $\arg \min _{z} \sum_{k=1}^{\left|S_{j}\right|} z_{k}\left\|\bar{b}+\bar{e}_{k}\right\|_{2}$ \\
\hline Local Ranking & $L_{r}\left(L_{r p}\right)$ & $\arg \min _{z} \sum_{k=1}^{\left|S_{j}\right|} z_{k}\left\|\bar{e}_{k}\right\|_{2}$ \\
\hline
\end{tabular}

Table 2: A summary of the optimization functions. Symbols between parentheses identify the optimization strategies that use the neighborhood pruning operation discussed in Sec. 6

\section{Neighborhood Pruning}

So far we considered only a sort of "blind" optimization, that can be largely improved in some specific, but not unrealistic, situations. Consider for instance the case of a peer $p_{j}$ that is placed on a leaf node of the underlay topology, and assume that its only underlay neighbor also contains a peer $p_{i}$. Does it 
make sense for $p_{j}$ to choose more than one neighbor in the overlay? Under a performance point of view it makes no sense, all the traffic that $p_{j}$ receives will pass through $p_{i}$. Under a robustness point of view it still makes sense, because $p_{i}$ may suddenly leave the overlay and $p_{j}$ would be logically disconnected. Yet it is intuitive to understand that it is convenient to reduce the neighborhood size of $p_{i}$, compared with another peer that resides in the center of the topology.

In general we can state that, regardless of the underlay topology, when $p_{j}$ selects its neighbors, if there exists a peer $p_{y}$ that lies on the shortest path between $p_{j}$ and another $p_{i}$, then $p_{y}$ should be preferred to $p_{i}$. In general, any node $p_{j}$ should prefer as neighbors the peers that are the first encountered along any shortest path route, because otherwise the data packets will "jump" some peer that are in the path to their destination, which is a waste of resources. This situation is not frequent if underlays are random graphs and if the number of peers is small compared to the number of underlay nodes, but it can be frequent, or even dominant, in realistic topologies with many leaf nodes and when the number of peers becomes comparable with the number of nodes in the underlay.

Based on the consideration above, we can re-define the objective of building an optimal overlay limiting the neighborhood selection to peers first encountered on shortest path routes. We call this operation (of limiting the selection) neighborhood pruning because the search for a neighbor along a branch is stopped immediately at the first peer encountered, and thus the branch is pruned.

The pseudocode of the steps executed by a generic peer $p_{j}$ for pruning the neighborhood are summarized in Algorithm 1 and detailed next.

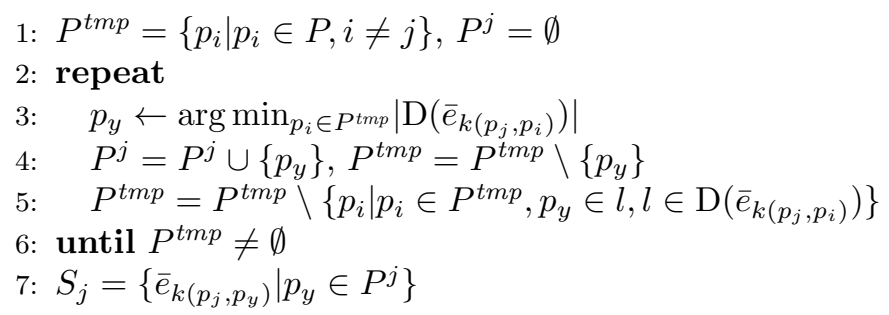

Algorithm 1: Neighbood pruning algorithm for peer $p_{j}$

In the initialization step (line 1) two sets are created: $P^{t m p}$ is initialized to $P \backslash\left\{p_{j}\right\}$ and is used to store the available peers; instead, $P^{j}$, initialized as an empty set, is used for storing the possible candidate neighbors of $p_{j}$. When a peer $p_{y}$ is selected as a candidate neighbor and inserted into $P^{j}$, all the other peers $p_{i}$ still available and which include $p_{y}$ in their shortest path from $p_{j}$ are removed from $P^{t m p}$ and not considered as possible candidate neighbors anymore. This is what happens in the main loop of the algorithm (lines 2-6): at the beginning of every iteration the peer $p_{y}$, which is the closest to $p_{j}$ among all the peers still available in $P^{t m p}$ (line 3 ), is inserted in $P^{j}$ and removed from $P^{t m p}$ (line 4). Finally, any peer $p_{i}$ which includes $p_{y}$ in its shortest path from $p_{j}$ is removed from $P^{t m p}$ (line 5 ). The loop stops when there are no more peers 
available $\left(P^{t m p}=\emptyset\right)$. After the loop, the algorithm builds the set $S_{j}$ used by the optimization strategies, which includes only the virtual links $\bar{e}_{k}$ that connect $p_{j}$ to the peers $p_{y}$ selected as candiate neighbors (line 7 ).

The computational complexity of the neighborhood pruning algorithm is linear in $|P|$ if we assume that the shortest paths computed by the Dijkstra functions are already available and that $p_{j}$ has enough memory to maintain a data structure for easily connecting every peer $p_{i}$ to the shortest paths that include $p_{i}$. If instead only the shortest paths computed by Dijkstra are available, the pruning algorithm is $O(|P| D)$, where $D$ is the diameter of the network. In any case, the neighborhood pruning algorithm can be easily integrated into the Dijkstra functions which could be modified for returning only the shortest paths originating from $p_{j}$ and terminating to the peers in $S^{j}$. Finally, note that when $\left|S^{j}\right| \leq d$ all the peers in $P^{j}$ are automatically selected as neigbors by $p_{j}$ without the need of executing any optimization.

The neighborhood pruning operation can be easily applied to all the local optimization strategies discussed in Sec. 5 with the difference that the scaling factor $s\left(e_{k}\right)$ introduced in Eq. (5) cannot be ignored, because every peer now potentially has a different degree. In particular, for computing the final performance metrics of the overlay, we set $s\left(e_{k}\right)=\left(d_{i} d_{j}\right) /\left(d_{i}+d_{j}\right)$, where $d_{i}, d_{j}$, computed with the knowledge of the local solutions of all the peers, are the real degrees of the endpoint peers of $e_{k}$. Instead, to compute the solution of the local optimization strategies, for each peer $p_{j}$, we set $s\left(e_{k}\right)=d_{j}$, where $d_{j}=\min \left(d,\left|S^{j}\right|\right)$. In this way the computational complexity is reduced because $p_{j}$ does not have to compute the pruned neighborhood of all the possible candidate neighbors in $S^{j}$.

\section{Results Evaluation}

We evaluate the proposed strategies implementing them in a simulator that computes the best overlay according to each strategy on synthetic network topologies. Simulations are performed using the Networkx library, a Python powerful library for the generation and analysis of graphs. Given an underlay topology we measure the load fairness of the generated overlay graph. The global and the local optimization problems are solved with the YAMLIP library, while the others are implemented directly in Python. We use three different kinds of underlay graphs, the well known Erdős-Rényi (ER), the Barabási-Albert (BA) model with preferential attachment, and a model from Cerdá-Alabern (CE) [39] derived from the analysis of a number of real mesh networks. The $\mathrm{CE}$ algorithm uses a preferential attachment algorithm to create a core of interconnected hosts, and then adds leaf hosts using a Gamma distribution. Finally we take advantage from real-life WCN topologies, taken from the Ninux and the FFWien ${ }^{4}$ networks [42] respectively made of 131 and 236 nodes.

\footnotetext{
${ }^{4}$ See http://ninux.org and http://www.funkfeuer.at/
} 


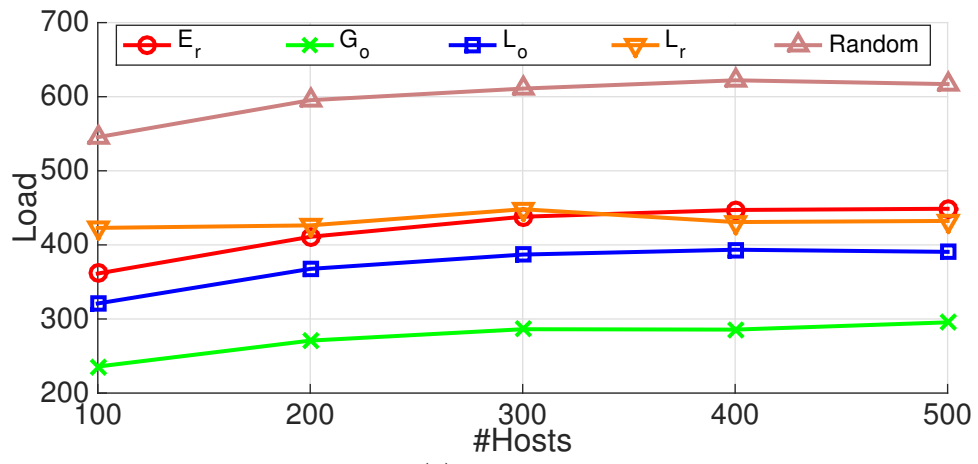

(a) Load for 20 peers

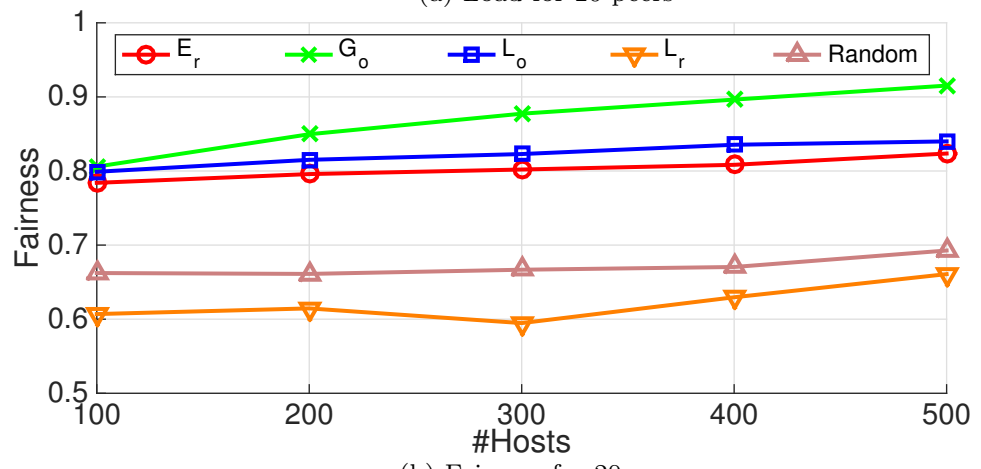

(b) Fairness for 20 peers

Figure 4: Load and fairness for an ER underlay from 100 to 500 hosts and 20 peers

\subsection{Results}

Fig. 4 reports the comparison of all the described strategies in a small (20 peers) scenario, increasing the size of an ER underlay. With 20 peers we are able to solve all the optimization problems, so this is a good benchmark to outline the differences between each strategy. The optimized results vastly outperform the random strategy, which is not surprising since they use available information on the underlay. What is most significant is that even the strategies that are less costly to compute, namely $L_{o}$ and $E_{r}$ achieve results that are close to the optimal strategy $G_{o}$ especially in terms of fairness.

This means that even if the quadratic optimization remains NP, in the analysed graphs the number of available disjoint paths between two peers is low and the space of the solutions of the optimization problem is small enough for all the optimization strategies to be very close. More results and discussions on this phenomenon can be found [1].

We performed experiments with overlay size increasing up to 100 peers, but with more than 20 peers the $G_{o}$ strategy is not solvable, so the comparison is done only with the relaxed strategies and their "pruned" version. Since there is no qualitative difference in the results we report only the results for 100 peers. Fig. 5 reports the results on a 100 peers overlay computed on CE underlays 


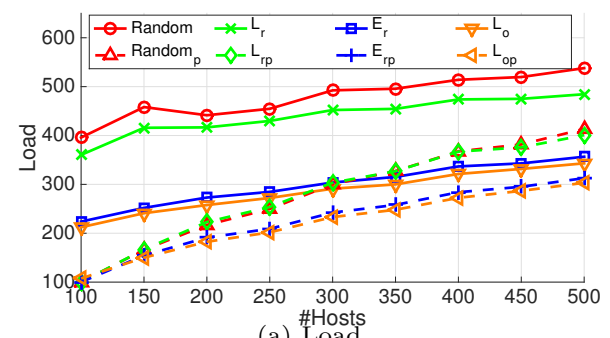

(a) Load

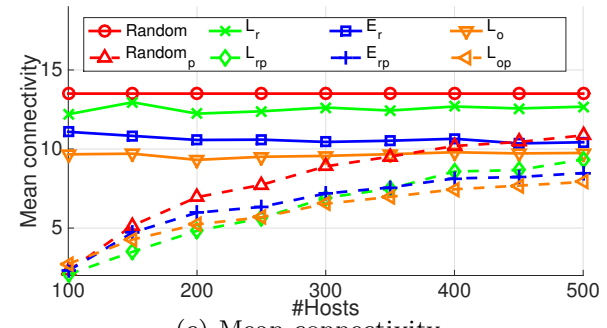

(c) Mean connectivity

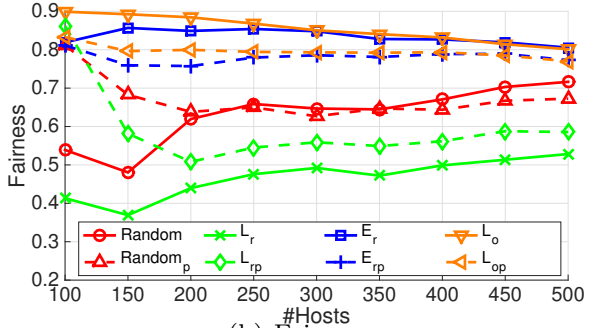

(b) Fairness

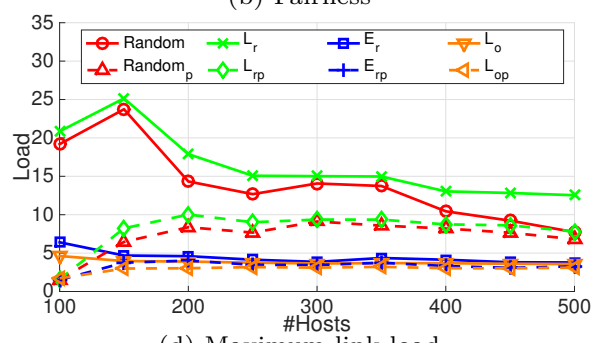

(d) Maximum link load

Figure 5: CE underlay, 100 peers, from 100 to 500 hosts

and shows two main results. The first is that the $L_{o}$ and $E_{r}$ strategies are very close and achieve a substantial improvement compared to the random strategy, which confirms the effectiveness of our approach. The $L_{r}$ strategy produces results that are closer to the random strategy. This is again an interesting result because it shows that the naive neighbor choice based only on distance $\left(L_{r}\right)$ produces a higher load than one can have if some long edges are added. Intuitively, choosing the peers that are the closest ones in the underlay seems the most reasonable solution. Instead, the shortest path between two peers computed on the underlay can be shorter than the sum of the shortest paths that pass through some other peers. The betweenness-based strategies try to avoid central peers which in some cases leads to the introduction of longer edges. These long edges may be network-wise more efficient than choosing neighbors only among the closest peers. The second important result is that every approach is substantially improved by the "pruning" strategy in terms of load on the underlay. This confirms that pruning neighbors is effective in excluding potential neighbors that are inherently inefficient.

If we look at the fairness, results are similar. Fig. $5 \mathrm{~b}$ shows that $L_{o}$ produces the highest fairness followed by $E_{r}$. They both fall in the $[0.8,0.9]$ range, which is extremely high. Random placement performs better than $L_{r}$. This confirms that the choice of the closest peer is not a good strategy because it stresses the peers (and the corresponding underlay hosts and links) that are more central. The pruned strategies lightly decrease the fairness due to a similar effect: pruning removes from $S_{j}$ the peers that are further away, thus it concentrates the choice on the closest peers. In this case the negative effect is very small. To further investigate this issue Fig. 5d shows the maximum load on any underlay link 


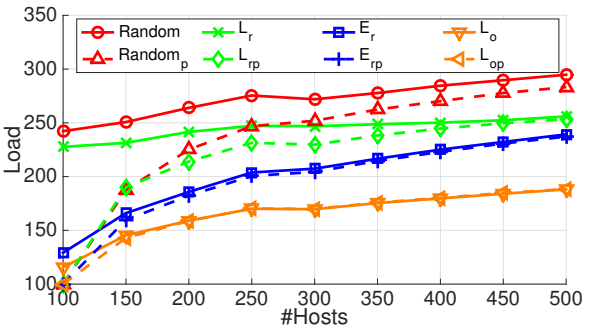

(a) Load

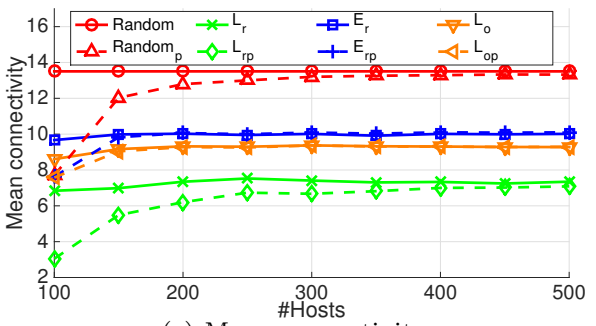

(c) Mean connectivity

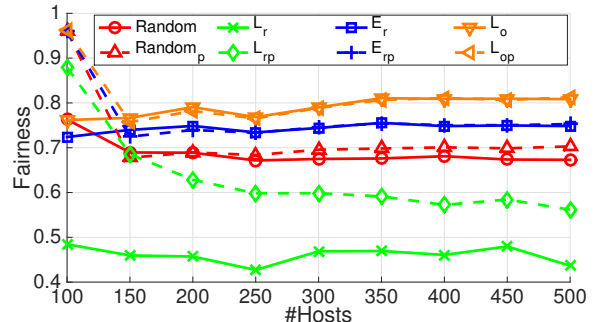

(b) Fairness

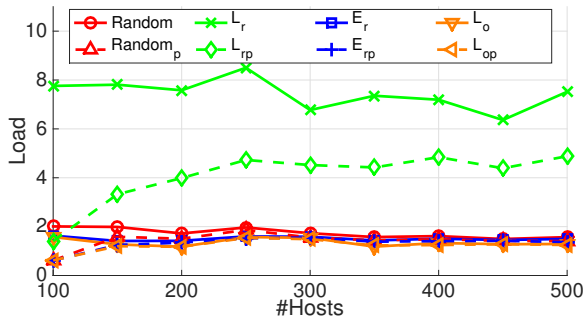

(d) Maximum link load

Figure 6: ER underlay, 100 peers, from 100 to 500 hosts

averaged on all the runs. The maximum load is reduced by pruning, so the load distribution, even if slightly less fair is more sustainable. Note also that, for an increasing number of hosts in the underlay, the maximum link load tends to keep a constant value or even decrease. This behavior is expected, in fact, when the number of hosts increases, the load of the underlay is distributed over a bigger number of links, thus reducing the maximum link load.

Finally in Fig. 5c we report the mean connectivity of the overlay computed by each strategy. The non-pruned strategies maintain a certain connectivity independently of the growth of the underlay. The pruned strategies instead increase the average connectivity with the growth of the underlay. To understand this effect, consider the point with 100 peers and 100 hosts. In this case the overlay corresponds to the underlay. The pruned strategies converge to almost the same average, because each peer can do nothing better than choosing as neighbors in the overlay the physical neighbors in the underlay. As the underlay grows the pruning algorithm leaves to each peer more neighbor candidates and thus, the average degree increases.

Fig. 6 and Fig. 7 report the same set of results computed on ER and BA topologies. The comparison among the non-pruned strategies confirms the same results we obtained in the $\mathrm{CE}$ topologies, the local optimization and the equalized ranking strongly improves both the random and the local ranking. This again confirms the effectiveness of our relaxation strategies. The effect of pruning is instead reduced. This is natural if we consider that by construction ER and BA underlays do not have leaf nodes, so the pruning effect is less visible. Indeed, even in this case, for the most dense scenarios there is a positive effect of pruning, which can be noticed in Fig. 8 where we report the load generated 


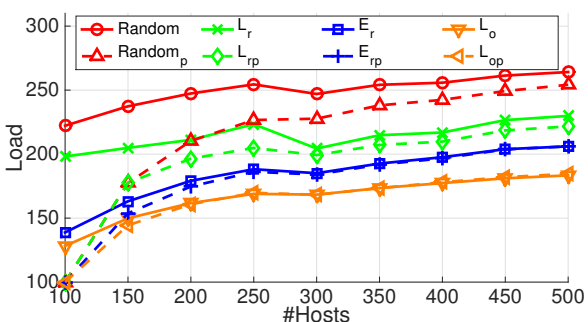

(a) Load

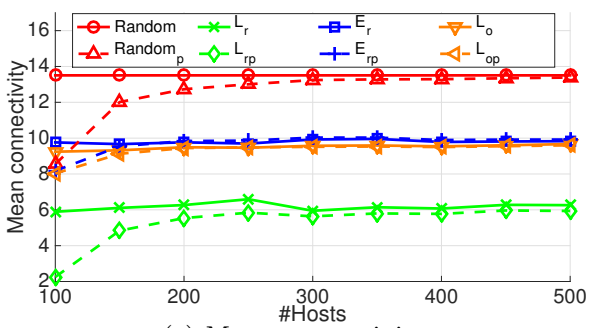

(c) Mean connectivity

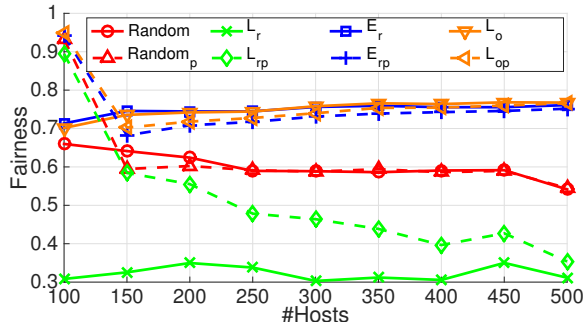

(b) Fairness

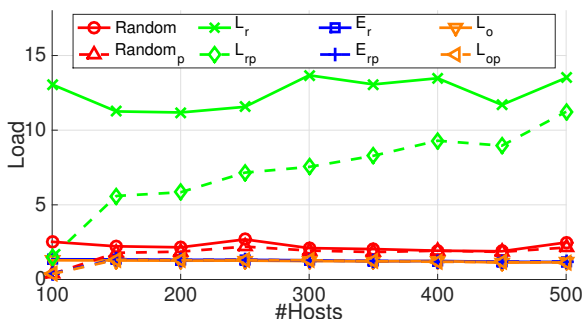

(d) Maximum link load

Figure 7: BA underlay, 100 peers, from 100 to 500 hosts

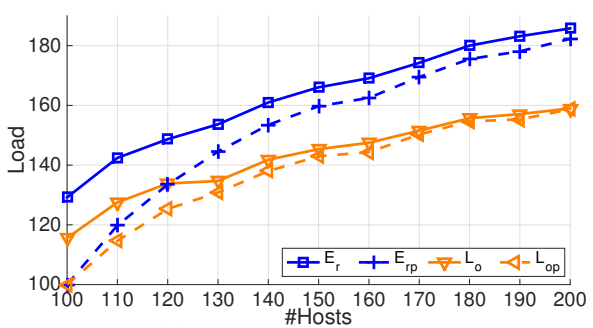

(a) Load for ER graphs

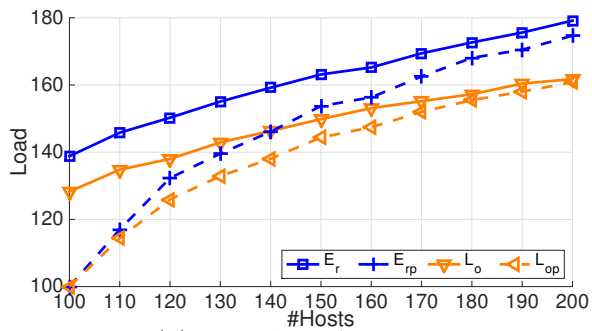

(b) Load for BA graphs

Figure 8: ER and BA underlay, 100 peers, from 100 to 200 hosts

by the two heuristic strategies, with and without pruning, in the range between 100 and 200 hosts. The figure shows that in dense overlays the improvement of pruning is still significant. As a general remark it also shows that it is difficult to optimize any distributed strategy for a generic network topology, and the the evaluation with realistic topologies (as we do with the CA topologies and with in Sec. 7.2) is essential to achieve sound results.

\subsection{Results on Real Topologies}

Figs. 9 and 10 report the results computed on two topologies derived from two real networks, the ninux and FunkFeuer networks, respectively made of 131 and 236 nodes. In this case, given the fixed underlay, we produced the results for an increasing size of the overlay which goes from a minimum of 30 peers to a maximum of 100 peers. The results are perfectly compatible with the ones obtained on the CE graphs. In real networks, where the number of leaf nodes 


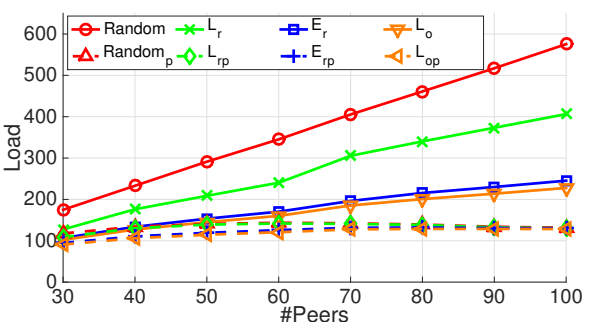

(a) Load

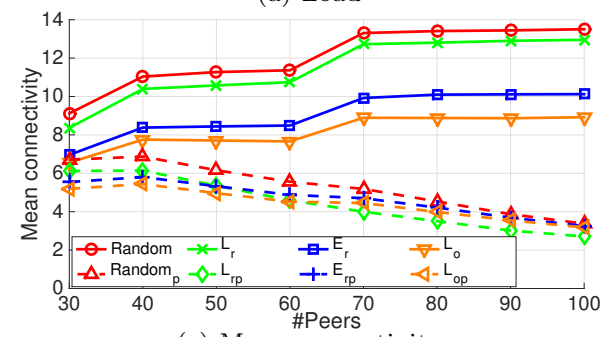

(c) Mean connectivity

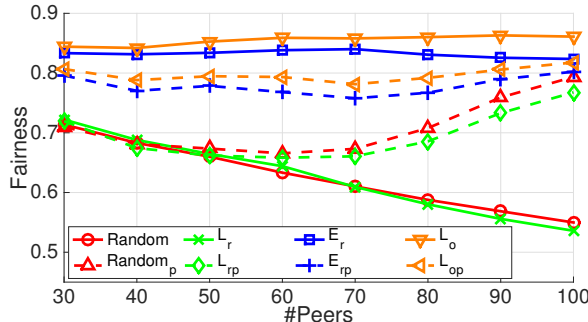

(b) Fairness

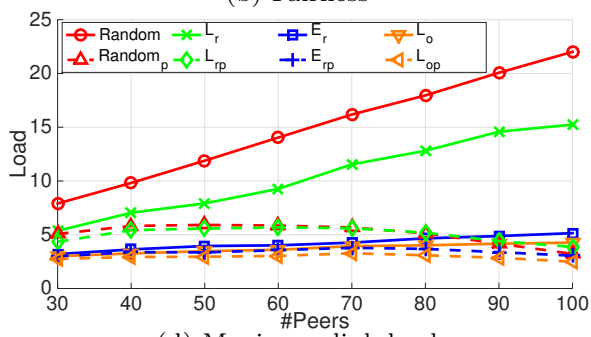

(d) Maximum link load

Figure 9: Ninux network, from 30 to 100 peers

is substantial, the pruned algorithms strongly improve the performance of the non pruned algorithms. As already shown for the CE underaly scenario, the improvement given by the "pruning" strategies in term of load on the underlay increases when the peers density increases (Figs. 9a and 10a), which confirms that the "pruning" is effective in excluding inefficient neighbors also in real network toplogies. This observation is confirmed also by the results reported in Figs. 9c and 10c that show how the mean connectivity decreases when the number of peers in the overlay becomes closer to the number of host in the underlay. In fact, in the scenario with 100 peers, most of them can do nothing better than choosing as neighbors in the overlay the physical neighbors in the underlay. This is not true for the case with 30 peers where the mean connectivity of the "pruning" strategies is more similar to the one obtained by the non-pruned strategies. Also with real network topologies the $E_{r p}$ and $L_{o p}$ strategies lightly decreases the fairness if compared with the corresponding non-pruned strategies, but the negative effect is very small and negligible from a practical point of view.

\section{Conclusions and Future Work}

Video streaming is one of the most popular Internet applications, thus achieving an efficient video streaming in a Wireless Mesh Network (WMN) with a small footprint on the precious wireless resources is of paramount importance. Starting from the observation that usually WMNs allow symmetrical bandwidth and a complete knowledge of the topology, we designed a novel strategy for cooperative (live) video streaming which can be successfully deployed on any multi-hop network that provides to the network nodes a complete view 


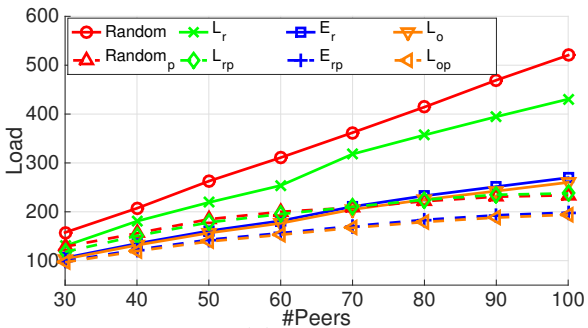

(a) Load

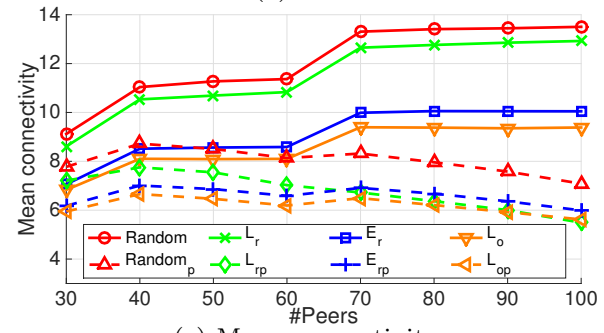

(c) Mean connectivity

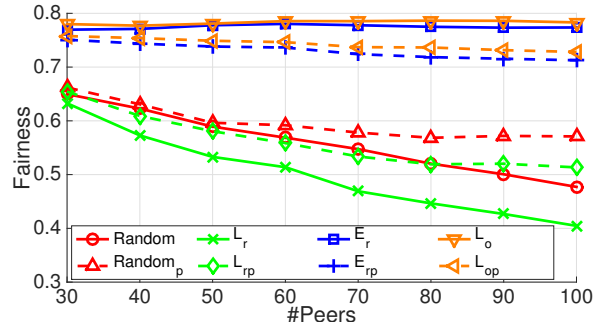

(b) Fairness

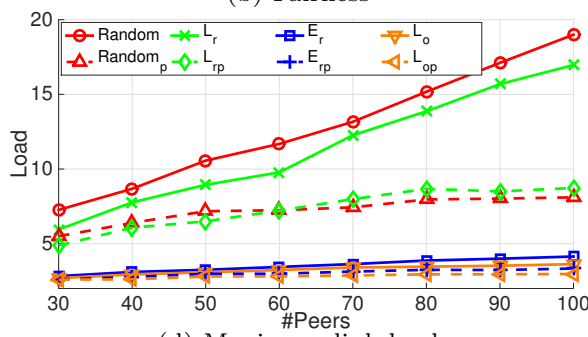

(d) Maximum link load

Figure 10: FFW network, from 30 to 100 peers

of the topology. Exploiting a novel mapping of the problem onto intersection graphs, we formulated an optimization problem to build an overlay that not only reduces the total load on the underlay, but also increases the fairness in the distribution of the load on the underlay links. Given the NP-completeness of the problem in its general formulation, we proposed two relaxations based on betweenness centrality. Simulation based results show that these strategies largely outperform random overlay building techniques. Even better performances can be obtained if the topological properties of real networks are taken into consideration, including a pruning strategy that takes into account the different topological positions of overlay nodes in the underlay.

Currently we have focused on underlays made of hundreds of hosts, so that the local solutions can be easily computed on low-power devices. However, heuristics introduced recently [43] make the computation of centrality metrics fast enough even on topologies made of thousands of nodes. As a further step, implementation in PeerStreamer is needed to allow the evaluation of the user experience and perceived quality with a real video streaming application. Furthermore, we will study the way to approximate the centrality of nodes even when the network uses a routing protocol that does not export all the network topology but just an approximation of it, such as a distance-vector protocol. Finally, we are also interested in evaluating how the proposed strategies can be applied to different application areas that face similar problems and would benefit from a more efficient use of the underlay (e.g., surveillance in urban sensor networks [44]). 


\section{References}

[1] L. Baldesi, L. Maccari, R. Lo Cigno, Improving P2P streaming in Wireless Community Networks, Computer Networks 93, Part 2 (2015) 389-403.

[2] D. Katsaros, N. Dimokas, L. Tassiulas, Social network analysis concepts in the design of wireless ad hoc network protocols, IEEE Network 24 (6) (2010) 23-29.

[3] L. Baldesi, L. Maccari, R. Lo Cigno, Optimized cooperative streaming in wireless mesh networks, in: 15th IFIP Networking Conference NETWORKING, Vienna, AT, May 2016, pp. 350-358.

[4] T. Clausen, P. Jaquet, Optimized link state routing protocol (OLSR), RFC 3626 (Oct. 2003).

[5] B. Braem, C. Blondia, C. Barz, H. Rogge, F. Freitag, L. Navarro, J. Bonicioli, S. Papathanasiou, P. Escrich, R. Baig Viñas, A. L. Kaplan, A. Neumann, I. Vilata i Balaguer, B. Tatum, M. Matson, A Case for Research with and on Community Networks, ACM SIGCOMM Comput. Commun. Rev. 43 (3) (2013) 68-73.

[6] M. Campista, P. Esposito, I. M. Moraes, L. Costa, O. Duarte, D. Passos, C. N. de Albuquerque, D. Saade, M. Rubinstein, Routing metrics and protocols for wireless mesh networks, IEEE Network 22 (1) (2008) 6-12.

[7] D. Pisinger, The quadratic knapsack problem-a survey, Discrete Applied Mathematics, Elsevier 155 (5) (2007) 623-648.

[8] R. Birke, E. Leonardi, M. Mellia, A. Bakay, T. Szemethy, C. Kiraly, R. Lo Cigno, F. Mathieu, L. Muscariello, S. Niccolini, J. Seedorf, G. Tropea, Architecture of a Network-Aware P2P-TV Application: The NAPA-WINE Approach, IEEE Communications Magazine 49 (2011) 154-163.

[9] N. Shah, D. Qian, An Efficient Unstructured P2P Overlay over MANET Using Underlying Proactive Routing, in: Seventh International Conference on Mobile Ad-hoc and Sensor Networks, 2011, pp. 248-255.

[10] D. Carra, R. Lo Cigno, E. W. Biersack, Graph Based Analysis of Mesh Overlay Streaming Systems, IEEE Jou. on Selected Areas in Communications 25 (9) (Dec. 2007) 1667-1677.

[11] G. Gheorghe, A. Montresor, R. Lo Cigno, Security and Privacy Issues in P2P Streaming Systems: A Survey, Springer Peer-to-Peer Networking and Applications 4 (Apr. 2011) 75-91.

[12] G. Ciccarelli, R. Lo Cigno, Collusion in Peer-to-Peer Systems, Elsevier Computer Networks 55 (15) (Oct. 2011) 3517-3532. 
[13] R. Fantacci, L. Maccari, T. Pecorella, Analysis of secure handover for IEEE 802.1x-based wireless ad hoc networks, IEEE Wireless Communications 14 (5) (2007) 21-29.

[14] A. J. Figueiredo Torres, E. Parente Ribeiro, C. M. Pedroso, Predictive delay-centric handover for video streaming over SCTP, Computer Communications 87 (2016) 49 - 59 .

[15] T. E. Ng, H. Zhang, Predicting internet network distance with coordinatesbased approaches, in: IEEE INFOCOM, New York, NY, USA, June 2002, pp. $170-179$.

[16] F. Dabek, R. Cox, F. Kaashoek, R. Morris, Vivaldi: A decentralized network coordinate system, ACM SIGCOMM Computer Communication Review 34 (4) (2004) 15-26.

[17] Y. Chen, X. Wang, C. Shi, E. K. Lua, X. Fu, B. Deng, X. Li, Phoenix: A weight-based network coordinate system using matrix factorization, Network and Service Management, IEEE Trans. on 8 (4) (2011) 334-347.

[18] R. Fortuna, E. Leonardi, M. Mellia, M. Meo, S. Traverso, QoE in Pull Based P2P-TV Systems: Overlay Topology Design Tradeoffs, in: 10th IEEE Int. Conf. on Peer-to-Peer Computing (P2P-10), Delft, NL, Aug. 2010, pp. 110 .

[19] R. Lo Cigno, A. Russo, D. Carra, On some fundamental properties of P2P push/pull protocols, in: IEEE ICCE 2008, Hoi An, Vietnam, June 2008, pp. 67-73.

[20] A. Russo, R. Lo Cigno, Delay-Aware Push/Pull Protocols for Live Video Streaming in P2P Systems, in: IEEE ICC, Cape Town, ZA, May 2010, pp. $1-5$.

[21] S. Traverso, L. Abeni, R. Birke, C. Kiraly, E. Leonardi, R. Lo Cigno, M. Mellia, Experimental comparison of neighborhood filtering strategies in unstructured P2P-TV systems, in: 12th IEEE Int. Conf. on Peer-to-Peer Computing (P2P-12), Tarragona, Spain, Sept. 2012, pp. 12-24.

[22] S. Traverso, L. Abeni, R. Birke, C. Kiraly, E. Leonardi, R. Lo Cigno, M. Mellia, Neighborhood Filtering Strategies for Overlay Construction in P2P-TV Systems: Design and Experimental Comparison, IEEE/ACM Trans. on Networking 23 (3) (2015) 741-754.

[23] S. Dolev, Y. Elovici, R. Puzis, Routing betweenness centrality, J. ACM 57 (4) (2010) 25:1-25:27.

[24] L. Maccari, R. Lo Cigno, Pop-routing: Centrality-based tuning of control messages for faster route convergence, in: 35th Annual IEEE Int. Conf. on Computer Communications (INFOCOM), San Francisco, CA, Apr. 2016, pp. 1-9. 
[25] L. Maccari, R. Lo Cigno, Waterwall: a cooperative, distributed firewall for wireless mesh networks, EURASIP Jou. on Wireless Communications and Networking 2013 (225) (Sept. 2013) 1-12.

[26] L. Maccari, R. Lo Cigno, Betweenness estimation in OLSR-based multi-hop networks for distributed filtering, Elsevier Jou. of Computer and System Sciences 80 (3) (May, 2014) 670-685.

[27] A. Vzquez-Rodas, L. J. de la Cruz Llopis, A centrality-based topology control protocol for wireless mesh networks, Ad Hoc Networks 24, Part B (2015) 34-54.

[28] L. Abeni, C. Kiraly, R. Lo Cigno, Robust Scheduling of Video Streams in Network-Aware P2P Applications, in: IEEE ICC 2010, Cape Town, ZA, May 2010, pp. 1-5.

[29] C. Kiraly, R. Lo Cigno, L. Abeni, Deadline-based Differentiation in P2P Streaming, in: IEEE Global Telecommunications Conference (GLOBECOM 2010), Miami, FL, Dec. 2010, pp. 1-5.

[30] Y. Sakata, K. Takayama, R. Endo, H. Shigeno, A Chunk Scheduling Based on Chunk Diffusion Ratio on P2P Live Streaming, in: IEEE NBiS, Sept. 2012, pp. $74-81$.

[31] K.-L. Hua, G.-M. Chiu, H.-K. Pao, Y.-C. Cheng, An efficient scheduling algorithm for scalable video streaming over P2P networks, Elsevier, Computer Networks 57 (14) (Oct. 2013) 2856-2868.

[32] J. Zhang, W. Xing, Y. Wang, D. Lu, Modeling and performance analysis of pull-based live streaming schemes in Peer-to-Peer network, Elsevier Computer Communications 40 (Mar. 2014) 22-32.

[33] Y. Liu, On the minimum delay peer-to-peer video streaming: how realtime can it be?, in: 15th Int. Conf. on Multimedia (ACM MULTIMEDIA '07), Augsburg, DE, Sept. 2007, pp. 127-136.

[34] L. Abeni, C. Kiraly, R. Lo Cigno, On the Optimal Scheduling of Streaming Applications in Unstructured Meshes, in: IFIP Networking, Aachen, DE, May 2009, pp. 117-130.

[35] J. Zhang, C. Yang, X. Zhang, A high-bandwidth live streaming model in mesh-based Peer-to-Peer networks, IEEE Communications Letters 20 (12) (2016) 2390-2393.

[36] F. Harary, Graph theory, Addison-Wesley, Reading, MA, 1969.

[37] H. D. Sherali, J. C. Smith, An improved linearization strategy for zero-one quadratic programming problems, Optimization Letters 1 (1) (2007) 33-47. 
[38] U. Brandes, C. Pich, Centrality estimation in large networks, Int. Jou. of Bifurcation and Chaos; Special Issue on Complex Networks' Structure and dynamics 17 (7) (2007) 2303-2318.

[39] L. Cerda-Alabern, On the topology characterization of Guifi.net, in: IEEE 8th Int. Conf. on Wireless and Mobile Computing, Networking and Communications (WiMob), 2012, pp. 389-396.

[40] R. Puzis, P. Zilberman, Y. Elovici, S. Dolev, U. Brandes, Heuristics for Speeding Up Betweenness Centrality Computation, in: Privacy, Security, Risk and Trust (PASSAT), Int. Conf. on and Int. Conf. on Social Computing (SocialCom), 2012, pp. 302-311.

[41] J. Lofberg, YALMIP: A toolbox for modeling and optimization in MATLAB, in: Computer Aided Control Systems Design, IEEE Int. Symposium on, IEEE, 2004, pp. 284-289.

[42] L. Maccari, R. Lo Cigno, A week in the life of three large Wireless Community Networks, Ad Hoc Networks 24, Part B, Elsevier (2015) 175-190.

[43] L. Maccari, Q. Nguyen, R. Lo Cigno, On the Computation of Centrality Metrics for Network Security in Mesh Networks, in: IEEE Global Communications Conf. (Globecom), Washington, DC, Dec. 2016, pp. 1-6.

[44] B. Rashid, M. H. Rehmani, Applications of wireless sensor networks for urban areas, J. Netw. Comput. Appl. 60 (C) (2016) 192-219. 